\title{
Dynamics of a Single Species in a Fluctuating Environment under Periodic Yield Harvesting
}

\author{
Mustafa Hasanbulli,, Svitlana P. Rogovchenko, ${ }^{2}$ and Yuriy V. Rogovchenko ${ }^{3,4}$ \\ ${ }^{1}$ Center for Theoretical Chemistry and Physics, New Zealand Institute for Advanced Study, Massey University Albany, \\ Auckland 0745, New Zealand \\ ${ }^{2}$ Department of Mathematics, Eastern Mediterranean University, Famagusta, TRNC, Mersin 10, Turkey \\ ${ }^{3}$ Department of Mathematical Sciences, University of Agder, Serviceboks 422, 4604 Kristiansand, Norway \\ ${ }^{4}$ Department of Mathematics and Mathematical Statistics, Umeå University, 90187 Umeå, Sweden
}

Correspondence should be addressed to Yuriy V. Rogovchenko; yuriy.rogovchenko@uia.no

Received 6 December 2012; Accepted 11 February 2013

Academic Editor: Theodore E. Simos

Copyright (C) 2013 Mustafa Hasanbulli et al. This is an open access article distributed under the Creative Commons Attribution License, which permits unrestricted use, distribution, and reproduction in any medium, provided the original work is properly cited.

\begin{abstract}
We discuss the effect of a periodic yield harvesting on a single species population whose dynamics in a fluctuating environment is described by the logistic differential equation with periodic coefficients. This problem was studied by Brauer and Sánchez (2003) who attempted the proof of the existence of two positive periodic solutions; the flaw in their argument is corrected. We obtain estimates for positive attracting and repelling periodic solutions and describe behavior of other solutions. Extinction and blow-up times are evaluated for solutions with small and large initial data; dependence of the number of periodic solutions on the parameter $\sigma$ associated with the intensity of harvesting is explored. As $\sigma$ grows, the number of periodic solutions drops from two to zero. We provide bounds for the bifurcation parameter whose value in practice can be efficiently approximated numerically.
\end{abstract}

\section{Introduction}

Environmental conditions like weather or food availability change significantly throughout the year and influence directly the growth of populations. Responding to seasonal environmental fluctuations, population density can alter quite fast during relatively brief periods, reflecting changes in the living conditions that become less favorable or converse. Since in many cases environmental fluctuations have a clearly pronounced seasonal character, they can be efficiently modeled with the help of nonautonomous differential equations with periodic coefficients. A striking example of a positive effect of a periodically fluctuating environment on the dynamics of a species has been reported by Jillson [1] who observed that total population numbers in the flour beetle population in the periodically fluctuating environment were more than twice those in the constant environment. On the other hand, Walters and Bandy [2] demonstrated positive effect of periodic harvesting concluding that periodic harvest of some big game populations may increase the total yield by
10 to 20 percent with the best interval between harvests in 2 to 4 years.

Although importance of the systematic study of the effect of environmental changes on the dynamics of populations has been emphasized in the monographs of MacArtur and Wilson [3], Nisbet and Gurney [4], Renshaw [5], Thieme [6], and other authors, many important problems remain open even for simple cases. As mentioned by Rosenblat [7, page 23], "seasonal and circadian changes in the surrounding conditions... can have a significant effect on birth and death rates, availability of resources, and so on. In spite of this, the question of the influence of these variations has received surprisingly little attention, certainly by comparison with the massive literature devoted to the analysis of systems in constant environments, and even by comparison with the studies of ecosystems in randomly fluctuating environments." In the introduction to the special issue of the journal Theoretical Population Biology "Understanding the role of environmental variation in population and community dynamics" (volume 64 (2003), issue 3), its editor Chesson [8] 
stressed that "the dominant focus in theoretical models of population and community dynamics has not been on how populations change in response to the physical environment, but on how populations depend on their own population densities or the population densities of other organisms."

In this paper, we investigate the effect of a periodic yield harvesting on the dynamics of a population in a fluctuating environment described by

$$
\frac{d x(t)}{d t}=r(t) x(t)\left(1-\frac{x(t)}{K(t)}\right),
$$

where the intrinsic growth rate $r$ and the carrying capacity of the environment $K$ are positive, continuous functions that vary periodically with time, $r(t+T)=r(t)$ and $K(t+T)=$ $K(t)$, for all $t \in \mathbb{R}$. Logistic equation (1) is widely used by ecologists, although its appropriateness as a model has been questioned, see Gabriel et al. [9]. Equation (1) does not describe correctly behavior of solutions if the condition $\bar{r} \stackrel{\text { def }}{=}(1 / T) \int_{0}^{T} r(t) d t>0$ fails to hold, see Rogovchenko and Rogovchenko [10]. Nevertheless, as Gabriel et al. [9, page 147] fairly noticed, "independently of the status that one gives to this model, it has been and remains a corner-stone of empirical and theoretical ecology."

The dynamics of harvested populations in a fluctuating environment has been addressed by several authors. We mention papers by Benardete et al. [11], Brauer and Sánchez [12], and Campbell and Kaplan [13] that stimulated the interest of the authors to the topic, as well as contributions by Lazer [14], Lazer and Sánchez [15, 16], and Liu et al. [17]. Contrary to proportional harvesting, the case where both $r$ and $K$ in (1) are periodic along with the harvesting term $H$ has been studied only by Brauer and Sánchez [12].

A bifurcation problem for a differential equation

$$
\frac{d x(t)}{d t}=k x(t)(1-x(t))-H(1+\sin (2 \pi t))
$$

has been discussed by Campbell and Kaplan [13] and, in more detail, by Benardete et al. [11], cf. [16, Example 1, page 157]. Unfortunately, argument developed by Benardete et al. [11] uses symmetry of the differential equation (2). Additional difficulties arise in case of variable coefficients because, as mentioned by Nkashama [18, page 2], "unlike the constant-coefficient case, the nonlinearity might have a string of non-zero $x$-intercepts in time." Recently, using CrandallRabinowitz saddle-node bifurcation theorem, Liu et al. [17] established existence of periodic solutions for $d x(t) / d t=$ $f(x(t))-\sigma h(t)$. However, neither they provide estimates for periodic solutions nor describe behavior of other solutions. As stressed by Padhi et al. [19, page 2617], "it would be interesting to develop results that identify the exact number of positive periodic solutions admitted by the considered model and study their stability nature. Such study becomes imperative from resource management perspective."

As Brauer and Sánchez [12, page 243] pointed out, "a general theory of the qualitative behavior of periodic population models, both single species and interacting species, would have many applications." In this paper, we obtain estimates for positive attracting and repelling periodic solutions to (1) in case of periodic yield harvesting, describe behavior of other solutions, and derive estimates for extinction and blowup times. This information is important for ecologists who can predict asymptotic behavior of solutions and evaluate their "life span." We also perform a detailed bifurcation analysis providing bounds for the bifurcation parameter $\sigma_{\text {bif }}$; these bounds can be tightened numerically. We are not, however, concerned with optimal harvesting policies; the reader is referred, for example, to the papers by Braverman and Mamdani [20], Castilho and Srinivasu [21], Fan and Wang [22], or $\mathrm{Xu}$ et al. [23]. Finally, we note that environmental fluctuations may be also modeled by including deviated arguments in logistic differential equations in a variety of ways, see, for instance, Gopalsamy [24], Zhang and Gopalsamy [25], Gopalsamy et al. [26, 27], and the references cited therein.

Remark 1. For obvious reasons, in population biology, only solutions that take on positive values should be taken into consideration. However, for completeness of mathematical analysis of the problem, we also investigate behavior of solutions that satisfy negative initial conditions or become negative at some instant $t_{*}$. In the former case, such analysis is completely irrelevant for applications, whereas in the latter case the phrase "solutions decay to $-\infty$ " should be interpreted in biological terms as "the population goes extinct"; we provide useful estimates for extinction times.

\section{Periodic Solutions and Harvesting}

2.1. Constant Yield Harvesting versus Proportional. We start by providing an introductory information regarding harvesting of a single species. In general case, harvesting of a population can be modeled by a differential equation

$$
\frac{d x(t)}{d t}=f(t, x(t))-h(t, x(t)),
$$

where the function $f$ describes the growth of unharvested population and the function $h$ provides a law according to which members of the population are removed. Two main harvesting options are described in the literature. A commonly used and widely studied type of harvesting where $h$ is a linear function of population size, $h(t, x(t))=H_{0} x(t)$, is known as proportional or constant effort harvesting. It often arises in mathematical models of fisheries under the assumption that the catch is proportional to the fishing effort $E$, see, for instance, the fundamental monograph of Clark [28]. The principal assumption that the catch is proportional to effort appears to be reasonable in many practical situations yet, it may be questionable for small or exhausted fisheries where much higher fishing effort should be required.

The type of harvesting where members of the population are removed at the constant rate per unit time, that is, $h(t, x(t))=H_{0}$, is called constant rate or constant yield harvesting. It arises in situations when a certain quota is specified (fishing or hunting licenses, etc.) and can be also described as regular harvesting at a stock-independent rate. It is reasonable to consider the case where $H_{0}$ is a function of 
time, $H_{0}=H_{0}(t)$; it can also be a periodic function. In a pioneering paper by Brauer and Sánchez [29], the case of logistic growth with a constant harvesting rate was considered. Since then, quite a few papers dealing with the harvesting of single and competing species have been published. However, as recently mentioned by the same authors [12, pages 233-234], "a plausible situation which has received little attention is when $H(t)$ is periodic, corresponding to seasonal harvesting such as seasonal open hunting or fishing seasons or crop spraying for parasites."

In addition to theoretical importance of the study undertaken in this paper, we also stress its practical importance. In fact, a logistic growth model with periodic harvesting

$$
\frac{d x(t)}{d t}=r(t) x(t)\left(1-\frac{x(t)}{K(t)}\right)-H(t),
$$

where $H(t)$ is a certain piecewise constant function with the period 12 has been used by Laham et al. [30] for the mathematical analysis of the best harvesting strategy for tilapia fish farming at selected fish farms in Malaysia. Furthermore, in the recent report by Keesom et al. [31], a differential equation similar to (2),

$$
\frac{d x(t)}{d t}=k x(t)\left(1-\frac{x(t)}{C}\right)-a(1+\sin (b t)),
$$

has been used for determination of an optimal harvesting frequency of fishing cycles $b$ required for maintaining a steady population of Alaskan salmon. Since both Keesom et al. [31] and Laham et al. [30] provide only numerical analysis for the models mentioned above, importance of a comprehensive theoretical analysis for this class of equations becomes obvious.

In what follows, we employ concepts of lower and upper fences, also termed lower and upper solutions (subsolutions and supersolutions). Basic facts regarding fences and funnels can be found in Hubbard and West [32, Chapters 1 and 4]. We interpret attractors and repellers using forward and pullback convergence, see Wiggins [33, page 112] and use the definition in Berger and Siegmund [34, Definition 3, page 3792], described by the authors as a "tailor-made specialization of more general concepts."

2.2. Proportional Harvesting. The case of periodic proportional harvesting,

$$
\frac{d x(t)}{d t}=r(t) x(t)\left(1-\frac{x(t)}{K(t)}\right)-H(t) x(t),
$$

where $H$ is a continuous positive periodic function has been studied by Castilho and Srinivasu [21, Proposition 3.2, page 6] and Fan and Wang [22, Theorem 4.1, page 172], cf. also Hale and Koçak [35, Exercise 4.23 on pages 128-129] and Sánchez [36, discussion on pages 884-885]. Clearly, (6) can be recast in the form (1) with a modified intrinsic growth rate $r_{1}(t)=r(t)-H(t)$ and carrying capacity $K_{1}(t)=(K(t)(r(t)-$ $H(t))) / r(t)$. Consequently, an application of results reported by Coleman et al. [37], Fan and Wang [22, Theorem 2.1, pages 167-168], or S. P. Rogovchenko and Y. V. Rogovchenko
[10, Theorem 13, page 1176] yields existence of a unique positive $T$-periodic solution $x_{p}(t)$ to (6) which is asymptotically stable with the domain of attraction containing positive initial data provided that the time average $\bar{r}_{1}$ of the function $r_{1}(t)$ is positive, $\bar{r}_{1}>0$.

2.3. Periodic Yield Harvesting. Compared to proportional harvesting, the case of periodic yield harvesting, (4) received much less attention. Results where at least one of the three functions $r, K$, or $H$ is constant are known, see, Braverman and Mamdani [20], Fan and Wang [22], Xu et al. [23]. To the best of our knowledge, (4) with all three periodic coefficients has been studied only by Brauer and Sánchez [12].

Contrary to (6), Riccati differential equation (4) does not have the trivial solution $x_{\text {triv }}(t) \equiv 0$, unless $H(t) \equiv 0$. This distinguishes dynamics of solutions with small positive initial data. Although any two solutions of (4) with $0<x_{1}\left(t_{0}\right)<$ $x_{2}\left(t_{0}\right)$ satisfy $x_{1}(t)<x_{2}(t)$, for all $t \geq t_{0}$, for any solution $x_{\text {small }}(t)$ of this equation with $0<x_{\text {small }}\left(t_{0}\right)<\varepsilon$, for a small $\varepsilon>0$, there exists a $t_{\text {ext }}$, termed the "extinction time," such that $x_{\text {small }}\left(t_{\text {ext }}\right)=0$ and $x_{\text {small }}(t)<0$, for all $t>t_{\text {ext }}$, see Section 2.5. This cannot happen for (6), for which the unstable solution $x_{\text {triv }}(t)$ acts as a nonporous lower fence.

Sánchez [38, page 959] mentioned that a minor modification of the result due to Pliss [39, Theorem 9.6, pages 102-103] yields existence of at most two periodic solutions to equations with the right-hand side quadratic with respect to $x$, cf. [40, Theorem on page 30]. Consequence of the celebrated Massera Theorem [41], see Brauer and Sánchez [12, Theorem 1, page 234] or Sánchez [40, Theorem on page 34], is often used for establishing existence of periodic solutions to equations with a continuous, $T$-periodic right-hand side. As Brauer and Sánchez [12, page 234] pointed out, this $T$-periodic solution is asymptotically stable. Thus, we concentrate our efforts on establishing bounds for positive periodic solutions and analyze behavior of other solutions, cf. Rizaner and Rogovchenko [42].

In the sequel, we use notation $g_{\min }=\min _{0 \leq t \leq T} g(t)$ and $g_{\max }=\max _{0 \leq t \leq T} g(t)$ and assume that at least one of the inequalities $r_{\min } \leq r_{\max }, K_{\min } \leq K_{\max }, H_{\min } \leq H_{\max }$ is strict. Completing the square on the right-hand side of (4) as in Brauer and Sánchez [12, Section 4, pages 241-242], one concludes that the slope $d x(t) / d t$ is negative for all $t \in \mathbb{R}$ provided that

$$
\gamma(t) \stackrel{\text { def }}{=} H(t)-\frac{r(t) K(t)}{4}>0,
$$

in which case $x(t) \rightarrow-\infty$ as $t \rightarrow+\infty$. Thus, (4) has no periodic solutions and the population goes extinct.

Passing to the case when (7) fails to hold, Brauer and Sánchez [12, Section 4, pages 241-242] reasoned as follows. Assuming that the inequality

$$
\gamma(t)<0
$$

holds for all $t \in \mathbb{R}$, they argued that, for all $t,\left.x^{\prime}\right|_{x=0}<$ $0,\left.x^{\prime}\right|_{x=K_{\max }}<0$ and $\left.x^{\prime}\right|_{x=K_{\max } / 2}>0$. However, one can easily construct counter examples where the latter inequality does not hold, although (8) is satisfied. In fact, consider (4) with 
$r(t)=4+\sin (2 \pi t), K(t)=5+2 \sin (2 \pi t)$ and $H(t)=$ $3+\sin (2 \pi t)$. Then, for all $t \in \mathbb{R}, \gamma(t) \leq-1 / 4$, and (8) is satisfied, but the derivative of solution of the given differential equation,

$$
\begin{aligned}
\left.x^{\prime}\right|_{x=K_{\max } / 2}= & \frac{7(4+\sin (2 \pi t))}{2} \times\left(1-\frac{7}{2(5+2 \sin (2 \pi t))}\right) \\
& -(3+\sin (2 \pi t)),
\end{aligned}
$$

changes sign infinitely many times on $\mathbb{R}$.

Theorem 2. Assume that condition

$$
H_{\max }<\frac{r_{\min } K_{\min }}{4}
$$

is satisfied. Then (4) has two positive periodic solutions, $x_{+}(t)$ and $x_{-}(t)$, which are the forward and pullback attractors, respectively, and, for all $t \in \mathbb{R}$,

$$
0<x_{-}(t)<\frac{K_{\min }}{2}<x_{+}(t)<K_{\max } .
$$

Proof. Observe first that [40, Theorem on page 30] yields that (4) cannot have more than two periodic solutions. Furthermore, for all $t \in \mathbb{R}$,

$$
\begin{aligned}
\left.\frac{d x(t)}{d t}\right|_{x(t)=K_{\max }} & =r(t) K_{\max }\left(1-\frac{K_{\max }}{K(t)}\right)-H(t) \\
& \leq r(t) K_{\max }\left(1-\frac{K_{\max }}{K_{\max }}\right)-H(t) \\
& =-H(t) \\
& <0 .
\end{aligned}
$$

On the other hand, by virtue of (10),

$$
\begin{aligned}
\left.\frac{d x(t)}{d t}\right|_{x(t)=K_{\min } / 2} & =r(t) \frac{K_{\min }}{2}\left(1-\frac{K_{\min }}{2 K(t)}\right)-H(t) \\
& \geq r_{\min } \frac{K_{\min }}{2}\left(1-\frac{K_{\min }}{2 K_{\min }}\right)-H_{\max } \\
& =\frac{r_{\min } K_{\min }}{4}-H_{\max } \\
& >0 .
\end{aligned}
$$

By [40, Theorem on page 34], there exists a periodic solution $x_{+}(t)$ of (4) satisfying $K_{\min } / 2<x_{+}(t)<K_{\max }$. Direction field and uniqueness arguments imply that this solution is a forward attractor for all solutions of (4) with initial data satisfying $K_{\min } / 2<x\left(t_{0}\right)<K_{\max }$.

Keeping in mind that a pullback attractor is a forward repeller, see Rasmussen [43, Remark 3.4, page 272], introduce a new variable $\tau=-t$. If $x(t)$ is a solution to (4), then $\tilde{x}(\tau)=x(-t)$ satisfies

$$
\frac{d \tilde{x}(\tau)}{d \tau}=-r(\tau) \tilde{x}(\tau)\left(1-\frac{\tilde{x}(\tau)}{K(\tau)}\right)+H(\tau) .
$$

Note first that, for $\tilde{x}(\tau)=0$, the slope $d \tilde{x}(\tau) / d \tau$ is positive for all $\tau \in \mathbb{R}, d \tilde{x}(\tau) /\left.d \tau\right|_{\tilde{x}(\tau)=0}=H(\tau)>0$. Furthermore, by virtue of (10),

$$
\begin{aligned}
\left.\frac{d \tilde{x}(\tau)}{d \tau}\right|_{\tilde{x}(\tau)=K_{\min } / 2} & =-r(\tau) \frac{K_{\min }}{2}\left(1-\frac{K_{\min }}{2 K(\tau)}\right)+H(\tau) \\
& \leq-r_{\min } \frac{K_{\min }}{2}\left(1-\frac{K_{\min }}{2 K_{\min }}\right)+H_{\max } \\
& =H_{\max }-\frac{r_{\min } K_{\min }}{4}<0 .
\end{aligned}
$$

Therefore, another application of [40, Theorem on page 34] yields the existence of a unique forward attractor of (14) as $\tau \rightarrow+\infty$. Correspondingly, there exists a unique positive pullback attractor $x_{-}(t)$ satisfying (11).

2.4. Sharper Estimates for Periodic Solutions. Rough preliminary estimates (11) for the attractor-repeller pair are further improved in this section. For $i=1,2$, let $\bar{\varphi}_{i}$ and $\bar{\psi}_{i}$ be equilibrium solutions to autonomous differential equations

$$
\begin{aligned}
& \frac{d \varphi(t)}{d t}=r_{\min } \varphi(t)\left(1-\frac{\varphi(t)}{K_{\min }}\right)-H_{\max }, \\
& \frac{d \psi(t)}{d t}=r_{\max } \psi(t)\left(1-\frac{\psi(t)}{K_{\max }}\right)-H_{\min } .
\end{aligned}
$$

Theorem 3. Assume that (10) holds for all $t \in \mathbb{R}$. Suppose also that

$$
\frac{2 H_{\min }}{H_{\max }} \leq \frac{r_{\max }}{r_{\min }} .
$$

Then, for the attractor-repeller pair $x_{+}(t), x_{-}(t)$ in Theorem 2, one has

$$
0<\bar{\psi}_{1}<x_{-}(t)<\bar{\varphi}_{1}, \quad \bar{\varphi}_{2}<x_{+}(t)<K_{\max } .
$$

If, in addition,

$$
K_{\max }<2 K_{\min }
$$

then

$$
\bar{\varphi}_{2}<x_{+}(t)<\bar{\psi}_{2}<K_{\max }
$$

Proof. By (11), it suffices to consider only the values of $x$ between 0 and $K_{\max }$. However, one can completely control the behavior of the right-hand side of (4) only for $0 \leq x \leq K_{\min }$, in which case the following estimates hold:

$$
\begin{aligned}
r_{\min } x(t)\left(1-\frac{x(t)}{K_{\min }}\right)-H_{\max } \\
\leq r(t) x(t)\left(1-\frac{x(t)}{K(t)}\right)-H(t) \\
\quad \leq r_{\max } x(t)\left(1-\frac{x(t)}{K_{\max }}\right)-H_{\min } .
\end{aligned}
$$


Equilibrium solutions to differential equations (16) and (17) are given by

$$
\begin{gathered}
\bar{\varphi}_{1,2}=\frac{K_{\min }}{2}\left(1 \mp \sqrt{1-\frac{4 H_{\max }}{r_{\min } K_{\min }}}\right), \\
\bar{\psi}_{1,2}=\frac{K_{\max }}{2}\left(1 \mp \sqrt{1-\frac{4 H_{\min }}{r_{\max } K_{\max }}}\right) .
\end{gathered}
$$

Condition (18) ensures that these four equilibria are ordered as follows:

$$
\bar{\psi}_{1}<\bar{\varphi}_{1}<\bar{\varphi}_{2}<\bar{\psi}_{2}
$$

Indeed, note first that $\bar{\varphi}_{1}<\bar{\varphi}_{2}<K_{\min }$. Then, by virtue of $H_{\min } /\left(r_{\max } K_{\max }\right) \leq H_{\min } /\left(r_{\min } K_{\min }\right) \leq H_{\max } /\left(r_{\min } K_{\min }\right)<$ $1 / 4$, we conclude that $\bar{\varphi}_{2}<\bar{\psi}_{2}$. To demonstrate that $\bar{\psi}_{1}<\bar{\varphi}_{1}$, we keep $H_{\max }, H_{\min }, r_{\max }$, and $r_{\min }$ fixed and analyze the behavior of $\bar{\varphi}_{1}\left(K_{\min }\right)$ and $\bar{\psi}_{1}\left(K_{\max }\right)$ described by the function $\chi(x)=x(1-\sqrt{1-v / x}) / 2, x \geq \nu$. One can verify that $\chi$ is strictly decreasing on $[v,+\infty)$, attains its maximal value $v / 2$ at $x=v$, and decays to $v / 4$ as $x \rightarrow+\infty$. Correspondingly, $\bar{\varphi}_{1}\left(K_{\min }\right) \in\left(H_{\max } / r_{\min }, 2 H_{\max } / r_{\min }\right]$ and $\bar{\psi}_{1}\left(K_{\max }\right) \in$ $\left(H_{\min } / r_{\max }, 2 H_{\min } / r_{\max }\right]$. Finally, condition (18) guarantees that $\max _{K_{\max }} \bar{\psi}_{1}\left(K_{\max }\right)=2 H_{\min } / r_{\max }<H_{\max } / r_{\min }=$ $\inf _{K_{\min }} \bar{\varphi}_{1}\left(K_{\min }\right)$, which completes the proof of (24). One has

Consider now the functions $\alpha_{1}(t)=\bar{\varphi}_{1}$ and $\beta_{1}(t)=\bar{\psi}_{1}$.

$$
\begin{aligned}
f\left(t, \alpha_{1}(t)\right) & =r(t) \bar{\varphi}_{1}\left(1-\frac{\bar{\varphi}_{1}}{K(t)}\right)-H(t) \\
& \geq r_{\min } \bar{\varphi}_{1}\left(1-\frac{\bar{\varphi}_{1}}{K_{\min }}\right)-H_{\max } \\
& =\alpha_{1}^{\prime}(t), \\
f\left(t, \beta_{1}(t)\right) & =r(t) \bar{\psi}_{1}\left(1-\frac{\bar{\psi}_{1}}{K(t)}\right)-H(t) \\
& \leq r_{\max } \bar{\psi}_{1}\left(1-\frac{\bar{\psi}_{1}}{K_{\max }}\right)-H_{\min } \\
& =\beta_{1}^{\prime}(t) .
\end{aligned}
$$

Hence, $\alpha_{1}(t)=\bar{\varphi}_{1}$ and $\beta_{1}(t)=\bar{\psi}_{1}$ are lower and upper fences, whereas the horizontal strip bounded by $\bar{\varphi}_{1}$ and $\bar{\psi}_{1}$ is an antifunnel, and there exists a periodic solution to (4) located between $\alpha_{1}(t)$ and $\beta_{1}(t)$. For the repeller $x_{-}(t)$, one has, for all $t \in \mathbb{R}, \bar{\psi}_{1}<x_{-}(t)<\bar{\varphi}_{1}$. If (20) is satisfied, one can show that both estimates for the attractor $x_{+}(t)$ are also "tightened" to (21). Otherwise, only a lower bound can be improved, which leads to (19).

Numerical values in the following example, as well as in the rest of the paper are truncated to four decimal places.

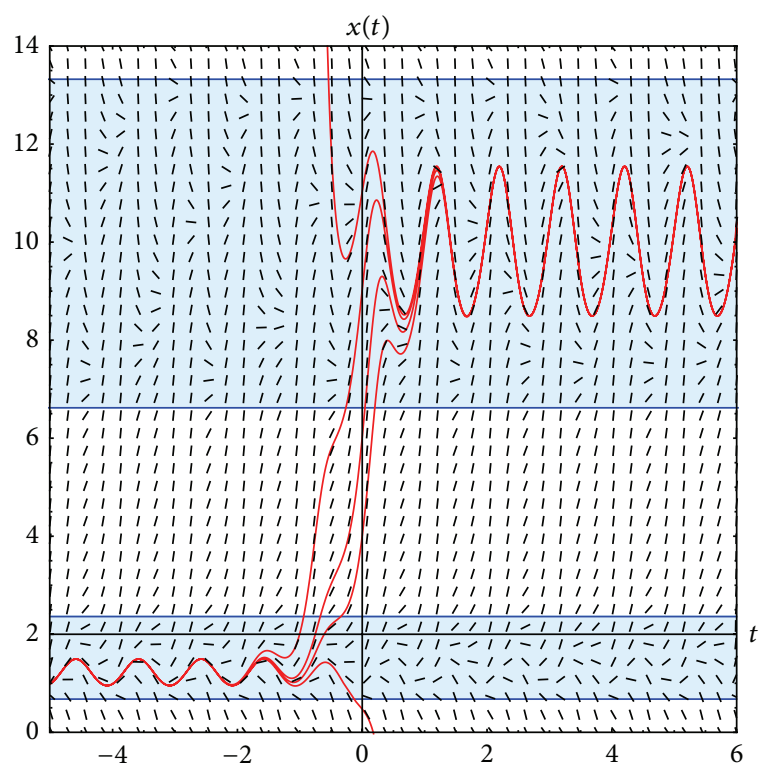

FIGURE 1: Tighter estimates for both periodic solutions of (26).

Example 4. Consider a 1-periodic differential equation

$$
\begin{aligned}
\frac{d x(t)}{d t}= & (5+\sin (2 \pi t)) x(t)\left(1-\frac{2 x(t)}{23+5 \cos (2 \pi t)}\right) \\
& -\frac{1}{2}(11-3 \cos (2 \pi t)) .
\end{aligned}
$$

In this case,

$$
\bar{\varphi}_{1,2}=\frac{3}{2}(3 \mp \sqrt{2}), \quad \bar{\psi}_{1,2}=7\left(1 \mp \frac{\sqrt{357}}{21}\right) .
$$

Condition (18) is satisfied because $2 H_{\min } / H_{\max }=2 \cdot 4 / 7 \leq$ $6 / 4=r_{\max } / r_{\min }$. By (19), $7(1-\sqrt{357} / 21)<x_{-}(t)<$ $3(3-\sqrt{2}) / 2<3(3+\sqrt{2}) / 2<x_{+}(t)<7(1+\sqrt{357} / 21)$, see Figure 1. The improvement achieved in comparison with rough estimates provided by Theorem 2 is seen as a light-colored "corridor" separating the funnel and antifunnel containing two periodic solutions. Corridor's width is given by $\bar{\varphi}_{2}-\bar{\varphi}_{1}=K_{\min } \sqrt{1-4 H_{\max } /\left(r_{\min } K_{\min }\right)}$; it equals $3 \sqrt{2}$ for (26). In addition, one can see two light-colored "corridors" of the same width $14-7(1+\sqrt{357} / 21)=7(1-\sqrt{357} / 21)=$ 0.7019 corresponding to improved upper and lower estimates for $x_{+}(t)$ and $x_{-}(t)$.

Remark 5. Exact solutions $\varphi(t)$ and $\psi(t)$ to (16) and (17) give tighter estimates for the solution $x(t)$ of (4) satisfying the same initial condition, provided that $0 \leq x\left(t_{0}\right)=\varphi\left(t_{0}\right)=$ $\psi\left(t_{0}\right) \leq K_{\min }$ or (20) holds. With the growth of $t$, both $\varphi(t)$ and $\psi(t)$ approach equilibria quite fast; even for relatively small values of $t$, the difference becomes hardly visible, see Figure 2. Thus, from the practical point of view, one can use estimates (19) and (21) excluding a small interval where solutions of (4) approach periodic ones as $t \rightarrow \mp \infty$. 


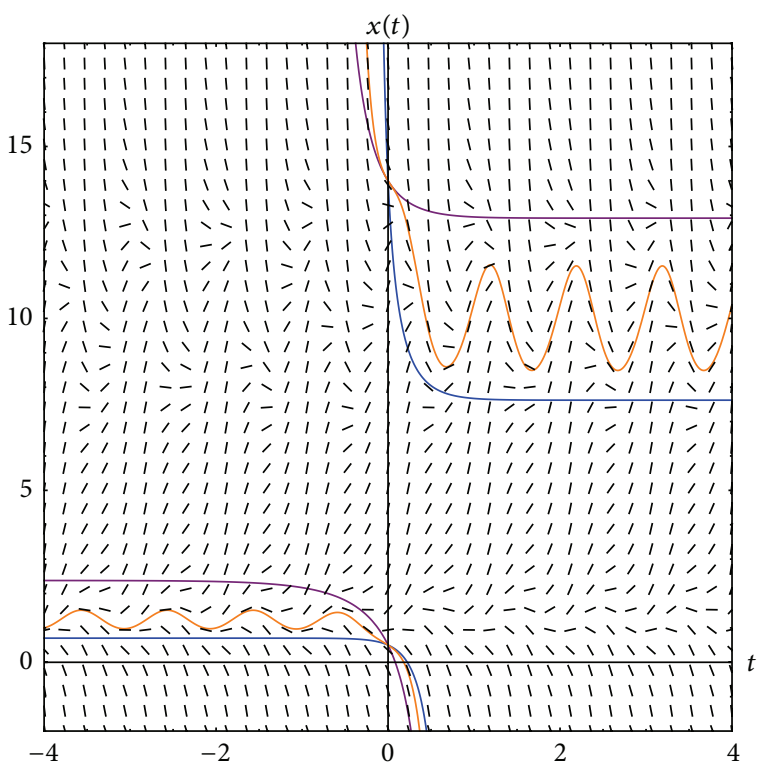

FIGURE 2: Unstable and stable periodic solutions embraced by upper and lower solutions.

Remark 6. To describe behavior of solutions $x(t)$ with initial data satisfying $x\left(t_{0}\right)<0$ or $x\left(t_{0}\right)>K_{\max }$, one needs estimates

$$
\begin{aligned}
r_{\max } x(t)\left(1-\frac{x(t)}{K_{\min }}\right)-H_{\max } \\
\quad \leq r(t) x(t)\left(1-\frac{x(t)}{K(t)}\right)-H(t) \\
\quad \leq r_{\min } x(t)\left(1-\frac{x(t)}{K_{\max }}\right)-H_{\min }
\end{aligned}
$$

rather than (22), because the first term on the right-hand side of (4) takes on negative values. Consequently, a pair of autonomous differential equations

$$
\begin{aligned}
& \frac{d \eta(t)}{d t}=r_{\max } \eta(t)\left(1-\frac{\eta(t)}{K_{\min }}\right)-H_{\max }, \\
& \frac{d \xi(t)}{d t}=r_{\min } \xi(t)\left(1-\frac{\xi(t)}{K_{\max }}\right)-H_{\min },
\end{aligned}
$$

is used instead of (16) and (17).

2.5. Extinction Times. We start with an upper bound for the extinction time $t_{\text {ext }}$ for solutions with initial data $x\left(t_{0}\right) \in$ $\left(0, K_{\min }\right]$. By (22), one has to study the behavior of solutions to differential equation (17). Suppose that

$$
H_{\min }>H_{\text {crit }}=\frac{r_{\max } K_{\max }}{4} .
$$

Note that (31) yields (7). Therefore, the slope defined by (17) is negative and all solutions of (4) decay to $-\infty$. Integrating (4), one derives the formula for the extinction time,

$$
\begin{aligned}
& t_{\text {ext }}^{s}=t_{0}+2 r_{\max }^{-1}\left(\frac{H_{\text {min }}}{H_{\text {crit }}}-1\right)^{-1 / 2} \\
& \times\left\{\tan ^{-1}\left(\frac{H_{\text {min }}}{H_{\text {crit }}}-1\right)^{-1 / 2}\right. \\
&+\tan ^{-1}\left(\frac{2}{K_{\max }}\left(\psi\left(t_{0}\right)-\frac{K_{\max }}{2}\right)\right. \\
&\left.\left.\times\left(\frac{H_{\text {min }}}{H_{\text {crit }}}-1\right)^{-1 / 2}\right)\right\} .
\end{aligned}
$$

In particular, if (20) holds, the extinction time for the solution $x_{*}(t)$ of (4) satisfying $x_{*}\left(t_{0}\right)=K_{\max } / 2$ is determined by a simpler expression,

$$
t_{\text {ext }}^{s}=t_{0}+2 r_{\max }^{-1}\left(\frac{H_{\min }}{H_{\text {crit }}}-1\right)^{-1 / 2} \tan ^{-1}\left(\frac{H_{\min }}{H_{\text {crit }}}-1\right)^{-1 / 2} .
$$

For solutions with initial data $x\left(t_{0}\right)>K_{\max }$, the situation is similar. Assume that (31) holds. By virtue of (28), differential equation (30) is used. Then, $H_{\max } \geq H_{\min }>H_{\text {crit }}=$ $r_{\max } K_{\max } / 4 \geq r_{\min } K_{\max } / 4=H_{*}$. Integrating (30), letting $\xi(t)=0$ and solving the resulting equation for $t_{\mathrm{ext}}^{l}$, one has

$$
\begin{aligned}
& t_{\text {ext }}^{l}=t_{0}+2 r_{\min }^{-1}\left(\frac{H_{\min }}{H_{*}}-1\right)^{-1 / 2} \times\left\{\tan ^{-1}\left(\frac{H_{\min }}{H_{*}}-1\right)^{-1 / 2}\right. \\
&+\tan ^{-1}\left(\frac{2}{K_{\max }}\left(\xi\left(t_{0}\right)-\frac{K_{\max }}{2}\right)\right. \\
&\left.\left.\times\left(\frac{H_{\min }}{H_{*}}-1\right)^{-1 / 2}\right)\right\} .
\end{aligned}
$$

Example 7. Consider a 1-periodic differential equation

$$
\begin{aligned}
\frac{d x(t)}{d t}= & \exp \left(\frac{1+\cos (2 \pi t)}{2}\right) \\
& \times x(t)\left(1-\frac{x(t)}{6+\cosh (\cos (2 \pi t))}\right) \\
& -(7+\sinh (\sin (2 \pi t))) .
\end{aligned}
$$




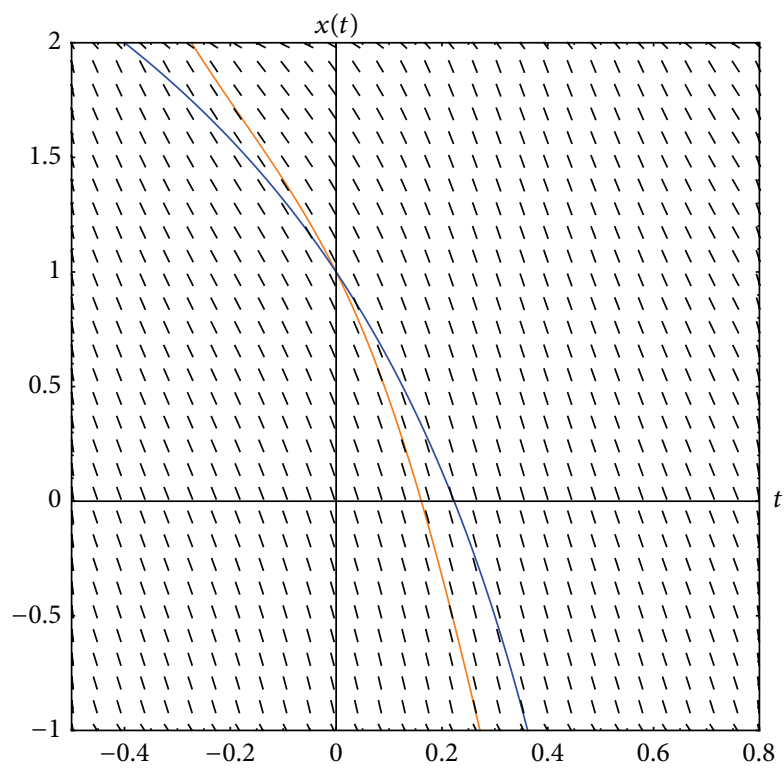

FIgURE 3: The upper solution (blue) and solution (orange) to (35) satisfying the initial condition $x(0)=1$. An upper bound for the extinction time for this solution is $t_{\mathrm{ext}}^{s}=0.2229$.

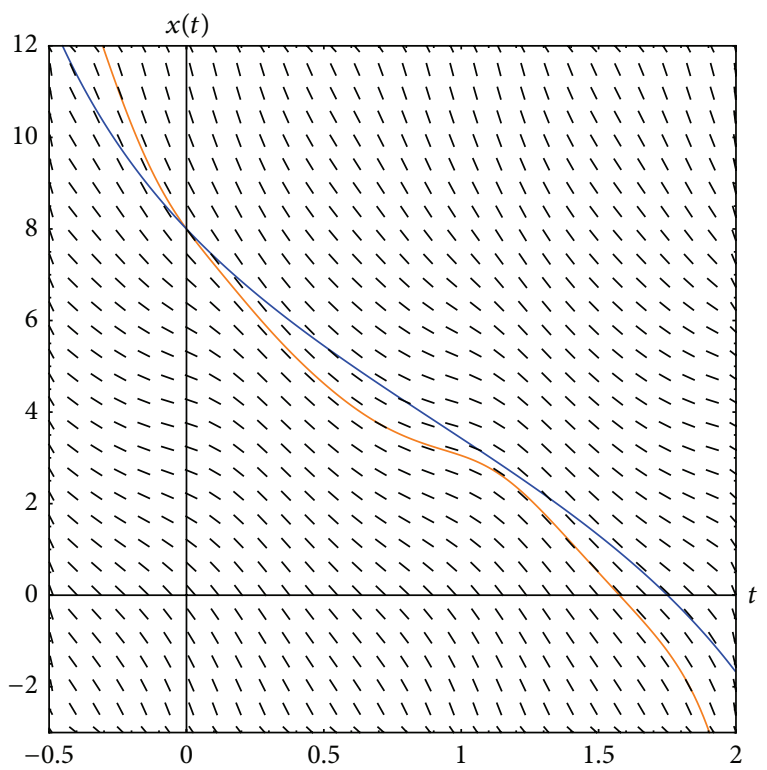

FIgURE 4: The upper solution (blue) and solution (orange) to (35) satisfying the initial condition $x(0)=8$. An upper bound for the extinction time for this solution is $t_{\mathrm{ext}}^{l}=1.7506$.

Condition (31) holds since $5.8248=H_{\min }>r_{\max } K_{\max } / 4=$ 5.1260 . Consequently, all solutions of (35) decay to $-\infty$. By (32), the extinction time for the solution satisfying $x(0)=$ $1<K_{\min }$ is $t_{\text {ext }}^{s}=0.2229$, see Figure 3. For a solution of (35) satisfying $x(0)=8>K_{\max }=6+\cosh 1=7.5431$, one has $8.1752=H_{\max }>r_{\min } K_{\max } / 4=1.8857$. By (34), an upper bound for the extinction time is $t_{\mathrm{ext}}^{l}=1.7506$, see Figure 4 .

2.6. Forward and Backward Blow-Up Time. In this section, we assume that (10) holds for all $t \in \mathbb{R}$. First, we estimate

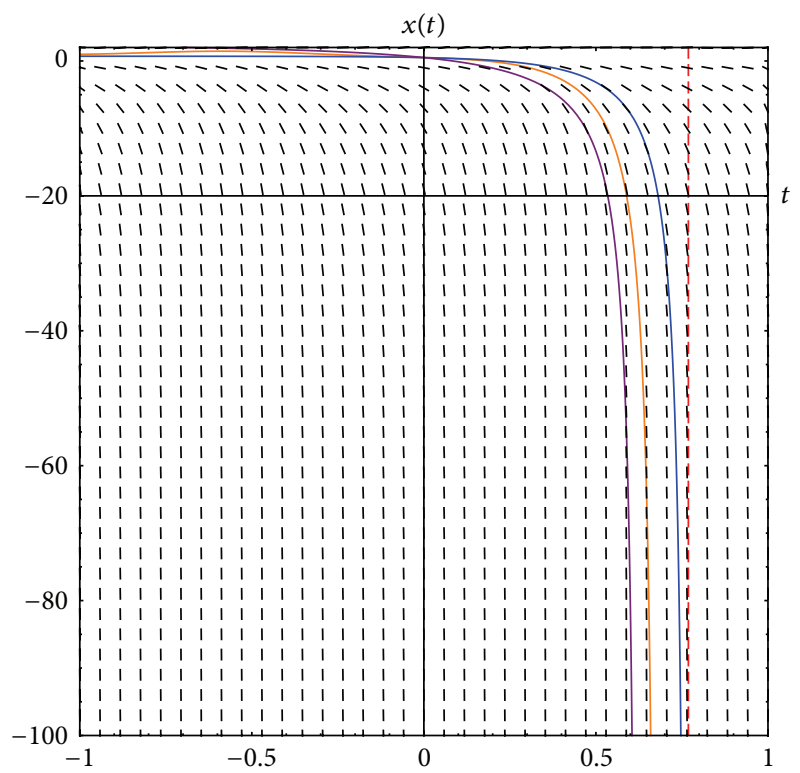

Figure 5: The upper solution (blue), lower (purple), and solution (orange) to (26), all satisfying the initial condition, $x(0)=0.5$, and the vertical asymptote for the upper solution.

forward blow-up time for "small" solutions with initial data $x\left(t_{0}\right) \in\left(0, \bar{\psi}_{1}\right)$, that is, for solutions located below the repeller $x_{-}(t)$. These solutions decay rapidly to $-\infty$ and have vertical asymptotes to the right of $t=t_{0}$. Taking into account estimates (22) that hold for small values of $x\left(t_{0}\right)$, we integrate differential equation (17) from $t_{0}$ to $t$ obtaining

$$
\psi(t)=\frac{\bar{\psi}_{1} c_{0} e^{\varkappa\left(t-t_{0}\right)}-\bar{\psi}_{2}}{c_{0} e^{\varkappa\left(t-t_{0}\right)}-1},
$$

where $c_{0}=\left(\psi\left(t_{0}\right)-\bar{\psi}_{2}\right) /\left(\psi\left(t_{0}\right)-\bar{\psi}_{1}\right), \varkappa=-r_{\max }\left(\bar{\psi}_{2}-\right.$ $\left.\bar{\psi}_{1}\right) / K_{\max }<0$, and equilibrium solutions $\bar{\psi}_{1}$ and $\bar{\psi}_{2}$ are defined above. By (36), solutions with small initial data $0<$ $x\left(t_{0}\right)<\bar{\psi}_{1}$ blow up in the future, $x(t) \rightarrow-\infty$ as $t \rightarrow t_{\text {forw }}^{-}$; an estimated value for a forward blow-up time $t_{\text {forw }}$ is given by

$$
t_{\text {forw }}=t_{0}+\frac{K_{\max } \ln \left(c_{0}^{-1}\right)}{r_{\max }\left(\bar{\psi}_{1}-\bar{\psi}_{2}\right)} .
$$

Equation $t=t_{\text {forw }}$ defines a vertical asymptote for solutions to (17); $t_{\text {forw }}>t_{0}$ because, for $x\left(t_{0}\right)<\psi\left(t_{0}\right)<\bar{\psi}_{1}$, one always has $c_{0}^{-1}<1$.

Example 8. To estimate a forward blow-up time for the solution to (26) satisfying the initial condition $x(0)=0.5$, note that equilibria of differential equation (17) associated with (26) are given by (27), $c_{0}=63.4036$. By virtue of (37), an upper bound for a forward blow-up time for the solution of (26) passing through the point $(0,0.5)$ is $t_{\text {forw }}=0.7686$, see Figure 5.

To estimate backward blow-up time for solutions to (4) with "large" initial data $x\left(t_{0}\right)>K_{\max }$ located above the attractor $x_{+}(t)$, we analyze asymptotic behavior of solutions using 


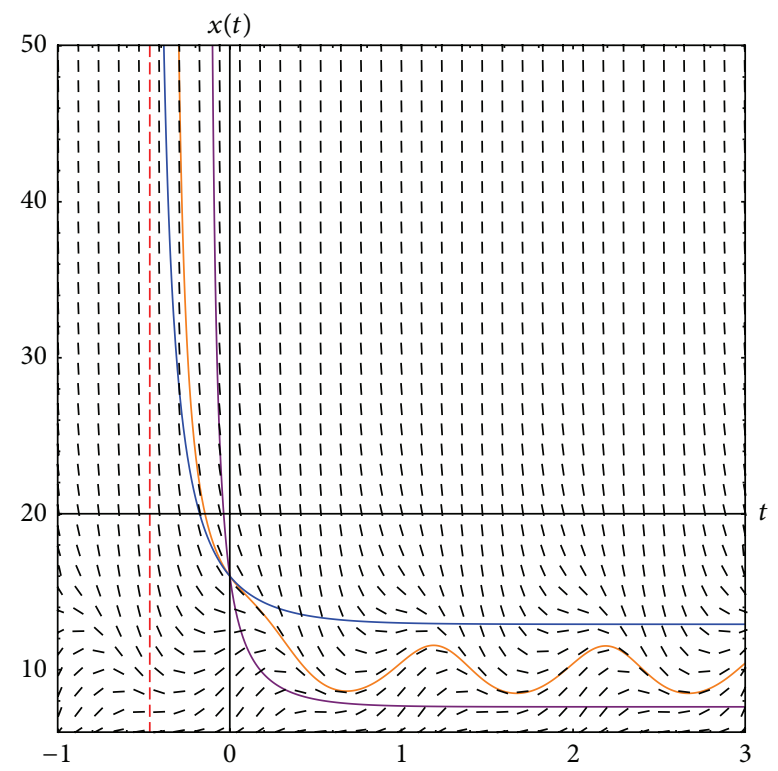

Figure 6: The upper (blue), lower (purple), and solution to (26) (orange), all satisfying the initial condition $x(0)=16$; a red dashed vertical asymptote for the upper solution is used for estimating a backward blow-up time.

inequalities (28) rather than (22). Integrating (30) from $t_{0}$ to $t$, one arrives at the formula

$$
t_{\mathrm{back}}=t_{0}+\frac{K_{\max } \ln \left(c_{0}^{-1}\right)}{r_{\min }\left(\bar{\xi}_{1}-\bar{\xi}_{2}\right)},
$$

where $c_{0}=\left(\xi\left(t_{0}\right)-\bar{\xi}_{2}\right) /\left(\xi\left(t_{0}\right)-\bar{\xi}_{1}\right), \varkappa=-r_{\min }\left(\bar{\xi}_{2}-\bar{\xi}_{1}\right) / K_{\max }<$ $0, \bar{\xi}_{1,2}=K_{\max }\left(1 \mp \sqrt{1-4 H_{\min } /\left(r_{\min } K_{\max }\right)}\right) / 2$, and $t_{\text {back }}<t_{0}$ since $c_{0}^{-1}>1$ for "large" initial data. Correspondingly, $x(t) \rightarrow$ $+\infty$ as $t \rightarrow t_{\text {back }}^{+}$.

Example 9. To find a backward blow-up time for a solution to (26) satisfying $x(0)=16>K_{\max }=14$, note that the equilibria of $(30)$ are $\bar{\xi}_{1}=7(1-\sqrt{35} / 7)=1.0839$ and $\bar{\xi}_{2}=7(1+$ $\sqrt{35} / 7)=12.9161$. Then, $c_{0}=0.2067$, and a lower bound for a backward blow-up time is estimated as $t_{\text {back }}=-0.4662$, see Figure 6. For an upper estimate for a backward blow-up time, note that equilibria for $(29)$ are $\bar{\eta}_{1}=9(1-\sqrt{39} / 9) / 2=1.3775$ and $\bar{\eta}_{2}=9(1+\sqrt{39} / 9) / 2=7.6225$. Then, $c_{0}^{*}=\left(\eta\left(t_{0}\right)-\right.$ $\left.\bar{\eta}_{2}\right) /\left(\eta\left(t_{0}\right)-\bar{\eta}_{1}\right)=0.5729$, and an upper bound for a backward blow-up time for a solution to (26) with $x(0)=16$ is $t_{\text {back }}^{*}=$ -0.1337 , see Figure 7.

Remark 10. In a similar manner, one can obtain two-sided estimates for extinction times derived in Section 2.5. Such estimates are useful for the evaluation of a "life span" of a given solution.

Remark 11. Nkashama [18, Theorem 2.1] established that solutions to

$$
\frac{d x(t)}{d t}=x(t)[a(t)-b(t) x(t)]
$$

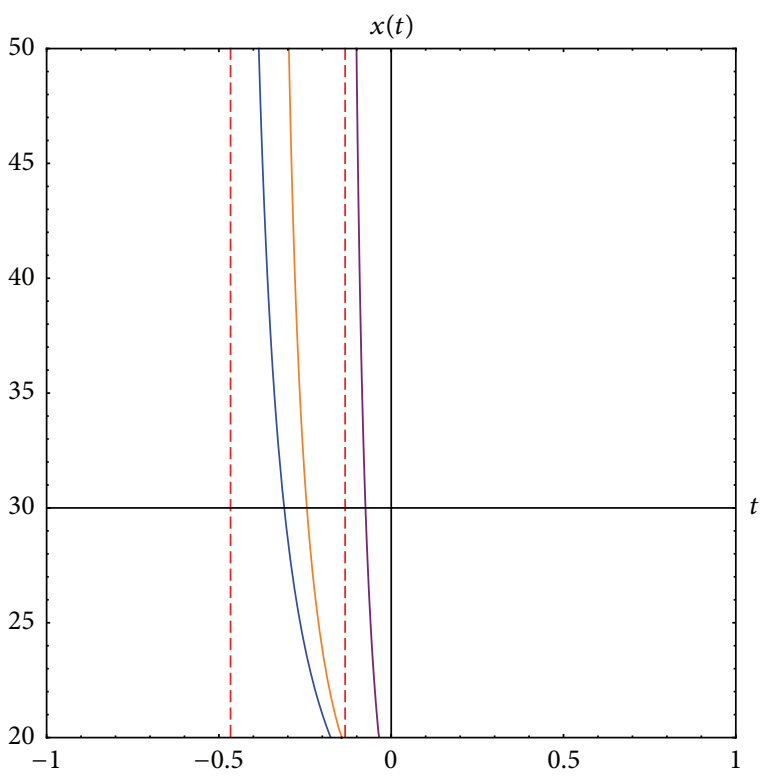

Figure 7: Red dashed lines represent two-sided estimates for backward blow-up time for a solution $x(t)$ (orange) of (26) with $x(0)=16$ squeezed between the upper (blue) and lower (purple) solutions.

located above the attractor $x_{+}(t)$ and below the repeller $x_{-}(t)$ blow up to $+\infty$ and $-\infty$, respectively, in finite time backward and forward. In our case, there exists a positive repeller for (4) instead of the trivial solution for (39). In addition, there exist positive solutions with small initial data that decay rapidly to $-\infty$, but do not have a vertical asymptote.

\section{Saddle-Node Bifurcation}

Consider now a $T$-periodic differential equation

$$
\frac{d y(t)}{d t}=r(t) y(t)\left(1-\frac{y(t)}{K(t)}\right)-\sigma H(t),
$$

where $\sigma>0$ is a parameter that characterizes the intensity of harvesting. An averaged system associated with (40) is

$$
\frac{d y(t)}{d t}=\bar{r} y(t)\left(1-\frac{y(t)}{\bar{K}}\right)-\sigma \bar{H}
$$

The change of variable $x=y-\bar{K} / 2$ reduces (41) to the form

$$
\frac{d x(t)}{d t}=a-b x^{2}(t)
$$

where $a=(\bar{r} \bar{K}) / 4-\sigma \bar{H}$ and $b=\bar{r} / \bar{K}$. Differential equation (42) with real parameters $a, b$ is the simplest canonical example of a saddle-node bifurcation with a nonhyperbolic equilibrium point at the origin. Consequently, one expects that (40) undergoes a so-called nonautonomous saddle-node bifurcation.

We know from Section 2.4 that, for $y \in\left[0, K_{\min }\right]$, (22) holds; for $y<0$ or $y>K_{\max },(28)$ is satisfied, whereas for $y \in\left(K_{\min }, K_{\max }\right)$, the sign of $r(t) y(1-y / K(t))$ cannot be 
controlled. Let $\lambda(t)=r(t) y(t)(1-y(t) / K(t))$. Denote by $I_{\text {neg }}$ the subset of points $t \in I_{T}=\left[t_{0}, t_{0}+T\right]$ such that $\lambda(t)<0$ for $t \in I_{\text {neg }}$. Then, for $y \in\left(0, K_{\max }\right)$, one has

$$
\begin{aligned}
\lambda(t) & -\sigma H(t) \\
& \leq \begin{cases}0, & \text { if } t \in I_{\text {neg }}, \\
r_{\max } y(t)\left(1-\frac{y(t)}{K_{\max }}\right)-\sigma H_{\min }, & \text { if } t \in I_{T} \backslash I_{\text {neg }} .\end{cases}
\end{aligned}
$$

Lemma 12. For

$$
\sigma>\sigma^{*}=\frac{r_{\max } K_{\max }}{4 H_{\min }},
$$

(40) has no periodic solutions; all solutions diverge to $-\infty$.

Proof. Let $y(t)$ be an arbitrary solution to (40). Taking into account that the function $g(y)=r_{\max } y\left(1-y / K_{\max }\right)$ attains its maximum value $r_{\max } K_{\max } / 4$ at $y=K_{\max } / 2$, we have, for all $t \in\left[t_{0}, t_{0}+T\right]$,

$$
\begin{aligned}
y\left(t_{0}\right. & +T)-y\left(t_{0}\right) \\
& =\int_{t_{0}}^{t_{0}+T}\left[r(t) y(t)\left(1-\frac{y(t)}{K(t)}\right)-\sigma H(t)\right] d t \\
& \leq \int_{I_{T} \backslash I_{\text {neg }}}\left[r_{\max } y(t)\left(1-\frac{y(t)}{K_{\max }}\right)-\sigma H_{\min }\right] d t \\
& \leq \int_{t_{0}}^{t_{0}+T}\left(\frac{r_{\max } K_{\max }}{4}-\sigma H_{\min }\right) d t \\
& =\left(\frac{r_{\max } K_{\max }}{4}-\sigma H_{\min }\right) T .
\end{aligned}
$$

If (44) is satisfied, one has $y\left(t_{0}+T\right)<y\left(t_{0}\right)$, and solution $y(t)$ cannot be periodic. Similarly, for any $n \in \mathbb{N}$, (44) yields

$$
\begin{aligned}
y\left(t_{0}\right. & +(n+1) T)-y\left(t_{0}+n T\right) \\
& \leq\left(\frac{r_{\max } K_{\max }}{4}-\sigma H_{\min }\right) T \\
& <0 .
\end{aligned}
$$

Therefore, for any solution $y(t)$ to $(40)$, one has $y(t) \rightarrow-\infty$ as $t \rightarrow+\infty$.

Remark 13. Condition (44) perfectly agrees with the assumption (7) that forces all solutions to decay to $-\infty$.

Theorem 2 assures the existence of the attractor-repeller pair for (40), for all

$$
\sigma<\sigma_{*}=\frac{r_{\min } K_{\min }}{4 H_{\max }} .
$$

To explore transition from the attractor-repeller pair to the case with no periodic solutions, we use nullclines and generalized nullclines.

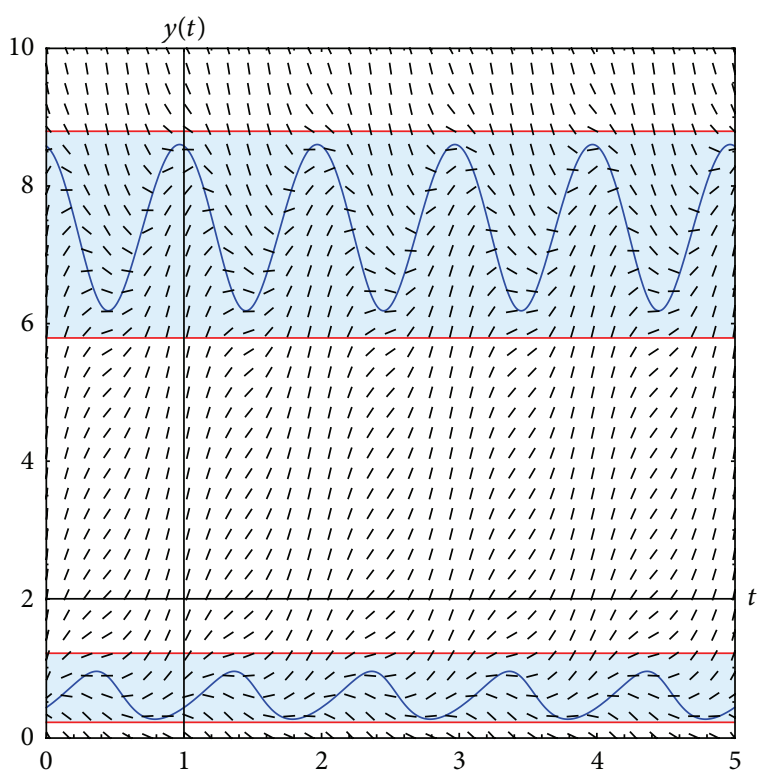

FIGURE 8: Nullclines and constant fences for (48) for $\sigma=1$.

Example 14. Consider a 1-periodic differential equation

$$
\begin{aligned}
\frac{d y(t)}{d t}= & (4+\cos (2 \pi t)) y(t)\left(1-\frac{y(t)}{8+\cos (2 \pi t)}\right) \\
& -\sigma(2+\sin (2 \pi t)) .
\end{aligned}
$$

Condition (47) holds for all $\sigma<7 / 4$. Equilibria for (16) and (17) are defined, respectively, by $\bar{\varphi}_{1,2}=7(1 \mp \sqrt{7(7-4 \sigma)} / 7) / 2$ and $\bar{\psi}_{1,2}=9(1 \mp \sqrt{5(45-4 \sigma)} / 15) / 2$. Taking in $(48) \sigma=1$, we obtain bounds for generalized nullclines $\zeta_{1}(t)$ and $\zeta_{2}(t), 9(1-$ $\sqrt{205} / 15) / 2<\zeta_{1}(t)<7(1-\sqrt{21} / 7) / 2<7(1+\sqrt{21} / 7) / 2<$ $\zeta_{2}(t)<9(1+\sqrt{205} / 15) / 2$, see Figure 8 .

Increasing the value of parameter $\sigma$ beyond $\sigma_{*}$, one observes that, at certain point, nullclines for (40) become pinched together, cf. Benardete et al. [11] or Campbell and Kaplan [13]. As the slope takes on negative values in regions between "trapping regions," some solutions can escape to $-\infty$.

Example 15. Consider a 1-periodic differential equation

$$
\begin{aligned}
\frac{d y(t)}{d t}= & (7-3 \sin (2 \pi t)) y(t)\left(1-\frac{y(t)}{10+2 \sin (2 \pi t)}\right) \\
& -3.8 \cdot(5+2 \cos (2 \pi t)) .
\end{aligned}
$$

Observe that $r_{\min } K_{\min } /\left(4 H_{\max }\right)=8 / 7<3.8=\sigma$. Generalized nullclines for (49) are shown in Figure 9.

Theorem 16. There exists a bifurcation value $\sigma_{\text {bif }}$ satisfying the inequality $r_{\min } K_{\min } /\left(4 H_{\max }\right) \leq \sigma_{\text {bif }} \leq r_{\max } K_{\max } /\left(4 H_{\min }\right)$ such that (40) has (i) no periodic solutions if $\sigma>\sigma_{\text {bif; }}$ (ii) exactly one periodic solution if $\sigma=\sigma_{\text {bif }}$; (iii) two periodic solutions if $\sigma<\sigma_{\text {bif }}$. 


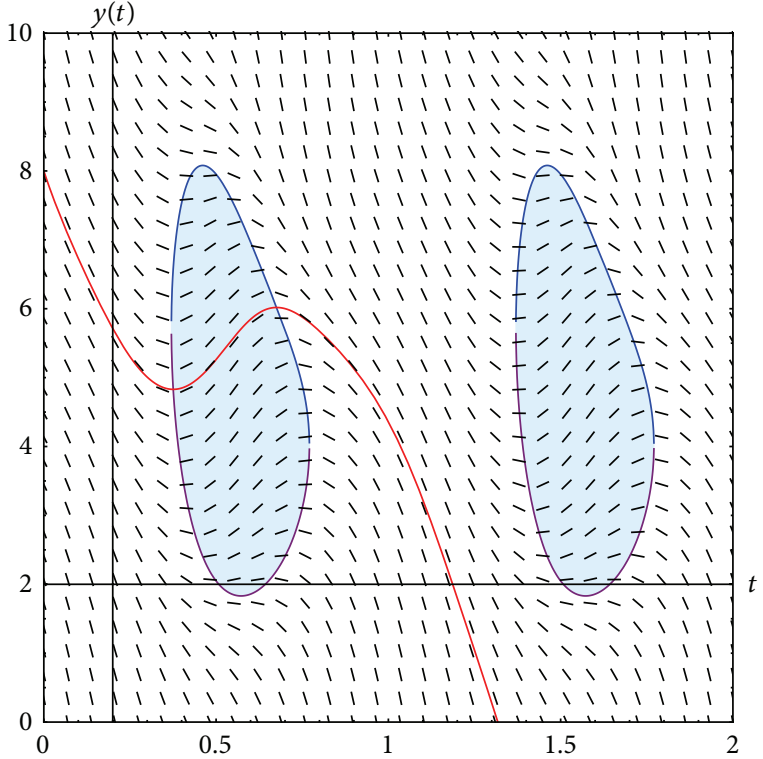

Figure 9: Pinched together pieces of upper (blue) and lower (purple) nullclines and one of solutions to (49) escaping the trapping region for $\sigma=3.8$.

Proof. Define the Poincaré map $h\left(y_{0}, \sigma\right)$ for $(40)$ on $(0, T)$. Equation $h\left(y_{0}, \sigma\right)=y_{0}$ is quadratic, see, for instance, [11, page 211]. Since the second derivative $h_{y y}\left(y_{0}, \sigma\right)$ is negative, the graph of $h\left(y_{0}, \sigma\right)$ is concave down. For $\sigma<r_{\min } K_{\min } / 4 H_{\max }$, it crosses the line $g\left(y_{0}\right)=y_{0}$ at two points corresponding to two periodic solutions of (40). On the other hand, the graph has no intersections with this line for $\sigma>r_{\max } K_{\max } / 4 H_{\min }$, and thus, in this case there are no periodic solutions to (40). The derivative $h_{\sigma}\left(y_{0}, \sigma\right)$ is negative. Hence, for each fixed $y_{0}$, the function $\delta(\sigma)=h\left(y_{0}, \sigma\right)$ decreases as $\sigma$ increases from $r_{\min } K_{\min } / 4 H_{\max }$ to $r_{\max } K_{\max } / 4 H_{\min }$, and there exists a unique value $\sigma=\sigma_{\text {bif }}$ such that the graph of $h\left(y_{0}, \sigma\right)$ is tangent to the line $g\left(y_{0}\right)=y_{0}$.

Remark 17. We stress that Benardete et al. [11, Section 5, pages 212-215] established bounds for the bifurcation parameter $\sigma_{\text {bif }}$ for a much simpler differential equation (2) using extensively its symmetry about the point $(1 / 4,1 / 2)$, whereas Theorem 16 requires no symmetry properties of (40) at all.

Example 18. Consider a 1-periodic differential equation

$$
\begin{aligned}
\frac{d y(t)}{d t}= & (5+\sin (2 \pi t)) y(t)\left(1-\frac{2 y(t)}{23+5 \cos (2 \pi t)}\right) \\
& -\frac{\sigma}{2}(11-3 \cos (2 \pi t)) .
\end{aligned}
$$

For $\sigma=1$, (50) turns into (26) for which the attractor-repeller pair exists, see Example 4 . One has $r_{\min } K_{\min } /\left(4 H_{\max }\right)=$ $9 / 7$ and $r_{\max } K_{\max } /\left(4 H_{\min }\right)=21 / 4$. By Theorem 16 , there exists a bifurcation value $\sigma_{\text {bif }} \in[9 / 7,21 / 4]$. Numerical experiments can be used to approximate the value of $\sigma_{\text {bif }}$. In fact, for $\sigma=2.395$, (50) has two periodic solutions plotted

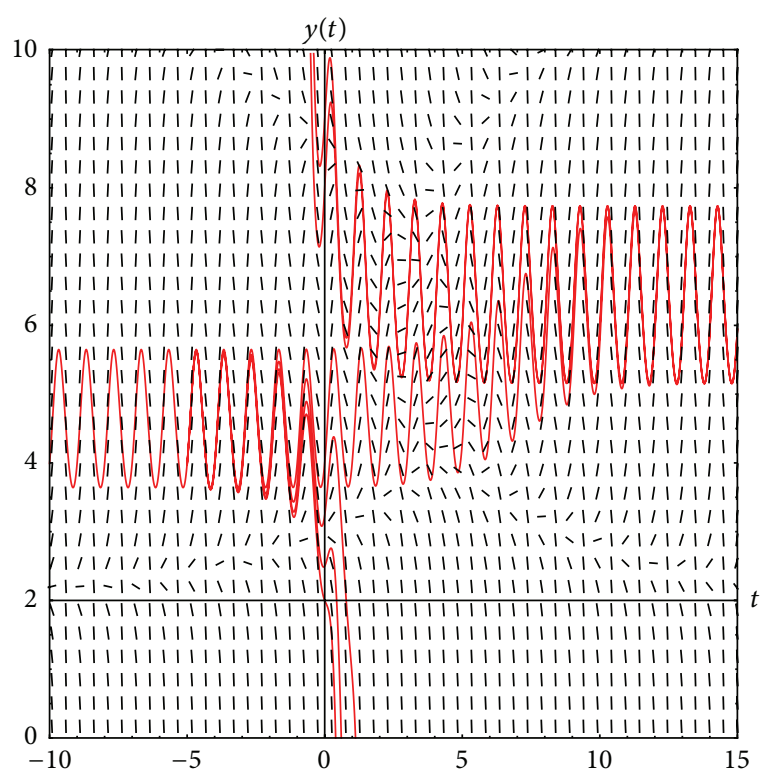

Figure 10: Several solutions to (50) for $\sigma=2.395$.

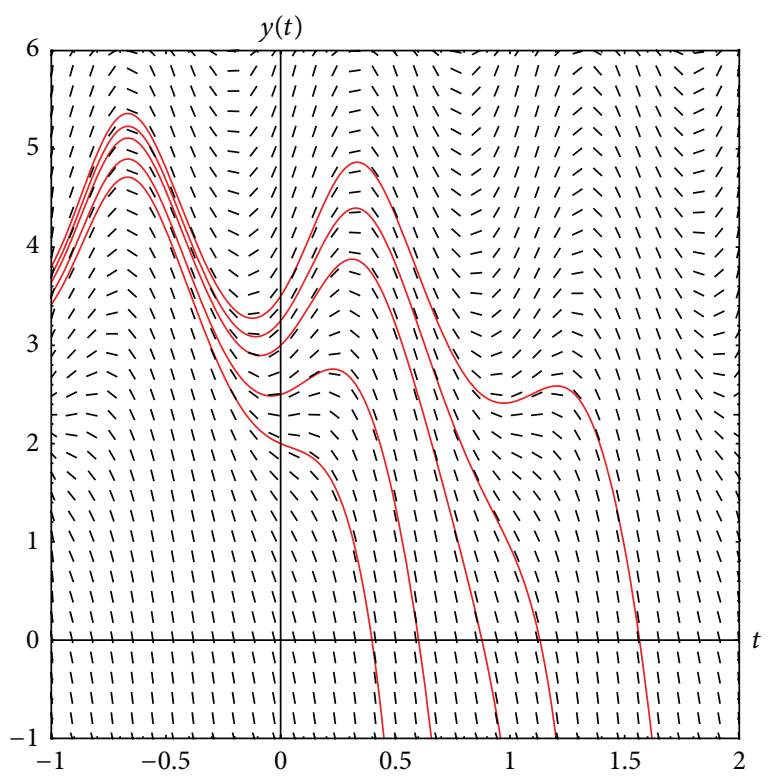

FIGURE 11: Several solutions to (50) for $\sigma=2.396$.

in Figure 10. On the other hand, for $\sigma=2.396$, (50) does not have periodic solutions anymore, see Figure 11. Consequently, $\sigma_{\text {bif }} \in(2.395,2.396)$.

\section{Conclusions}

Differential equation (4) exhibits quite interesting dynamics. Existence of the attractor-repeller pair is assured by condition (10); efficient estimates for periodic solutions are derived in Section 2.4. In the presence of the positive attractor-repeller pair, all other solutions to (4) fall into one of the three groups. Namely, as time $t$ advances, (i) solutions with small initial data $x\left(t_{0}\right) \in\left(0, x_{-}\left(t_{0}\right)\right)$ move away from $x_{-}(t)$, decay rapidly 
to $-\infty$, and blow up in the future as $t \rightarrow t_{\text {forw }}^{-}$(approach the repeller as $t \rightarrow-\infty$ ); (ii) solutions with initial data $x\left(t_{0}\right) \in\left(x_{-}\left(t_{0}\right), x_{+}\left(t_{0}\right)\right)$ leave the vicinity of the repeller $x_{-}(t)$ and approach the attractor $x_{+}(t)$ (correspondingly, leave the vicinity of the attractor $x_{+}(t)$ and approach the repeller $x_{-}(t)$ as $t \rightarrow-\infty$ ); these are so-called heteroclinic orbits; (iii) solutions with initial data $x\left(t_{0}\right)>x_{+}\left(t_{0}\right)$ approach the attractor $x_{+}(t)$, and blow up back in time as $t \rightarrow t_{\text {back }}^{+}$. If condition (7) holds, (4) has no periodic solutions. All solutions decay to $-\infty$; extinction time is estimated for solutions with small and large initial data.

Qualitative properties of (40) vary with the parameter $\sigma$. As $\sigma$ increases, the number of periodic solutions changes from two to zero; differential equation (40) undergoes a nonautonomous saddle-node bifurcation. Estimates for the bifurcation parameter $\sigma_{\text {bif }}$ can be significantly tightened by using numerical methods. Contrary to [11], our bifurcation analysis does not require symmetry of (40). We conclude by noting that although all numerical experiments in this paper were performed using Wolfram Mathematica 7.0, any computer algebra system can be used instead.

\section{Acknowledgments}

This research started when S. P. Rogovchenko and Yu. V. Rogovchenko visited the Abdus Salam International Centre for Theoretical Physics, Trieste, Italy, whose warm hospitality, excellent research facilities, and financial support are gratefully acknowledged. Yu. V. Rogovchenko also acknowledges the research grant from the Faculty of Science and Technology of Umeà University. The authors thank an anonymous referee for useful remarks that helped us to accentuate the importance of the study undertaken in this paper.

\section{References}

[1] D. A. Jillson, "Insect populations respond to fluctuating environments," Nature, vol. 288, no. 5792, pp. 699-700, 1980.

[2] C. J. Walters and P. J. Bandy, "Periodic harvest as a method of increasing big game yields," The Journal of Wildlife Management, vol. 36, pp. 128-134, 1972.

[3] R. MacArtur and E. O. Wilson, The Theory of Island Biogeography, Princeton University Press, Princeton, NJ, USA, 1973.

[4] R. M. Nisbet and W. S. C. Gurney, Modelling Fluctuating Populations, John Wiley \& Sons, New York, NY, USA, 1982.

[5] E. Renshaw, Modelling Biological Populations in Space and Time, vol. 11 of Cambridge Studies in Mathematical Biology, Cambridge University Press, Cambridge, UK, 1991.

[6] H. R. Thieme, Mathematics in Population Biology, Princeton Series in Theoretical and Computational Biology, Princeton University Press, Princeton, NJ, USA, 2003.

[7] S. Rosenblat, "Population models in a periodically fluctuating environment," Journal of Mathematical Biology, vol. 9, no. 1, pp. 23-36, 1980.

[8] P. Chesson, "Understanding the role of environmental variation in population and community dynamics," Theoretical Population Biology, vol. 64, no. 3, pp. 253-254, 2003.

[9] J. P. Gabriel, F. Saucy, and L. F. Bersier, "Paradoxes in the logistic equation?” Ecological Modelling, vol. 185, no. 1, pp. 147-151, 2005.
[10] S. P. Rogovchenko and Yu. V. Rogovchenko, "Effect of periodic environmental fluctuations on the Pearl-Verhulst model," Chaos, Solitons and Fractals, vol. 39, no. 3, pp. 1169-1181, 2009.

[11] D. M. Benardete, V. W. Noonburg, and B. Pollina, "Qualitative tools for studying periodic solutions and bifurcations as applied to the periodically harvested logistic equation," American Mathematical Monthly, vol. 115, no. 3, pp. 202-219, 2008.

[12] F. Brauer and D. A. Sánchez, "Periodic environments and periodic harvesting," Natural Resource Modeling, vol. 16, no. 3, pp. 233-244, 2003.

[13] D. Campbell and S. R. Kaplan, "A bifurcation problem in differential equations," Mathematics Magazine, vol. 73, no. 3, pp. 194-203, 2000.

[14] A. C. Lazer, "Qualitative studies of the solutions of the equation of population growth with harvesting," Matemáticas: Enseñanza Universitaria, vol. 17, pp. 29-39, 1980.

[15] F. Brauer and D. A. Sánchez, "Constant rate population harvesting: equilibrium and stability," Theoretical Population Biology, vol. 8, no. 1, pp. 12-30, 1975.

[16] A. C. Lazer and D. A. Sánchez, "Periodic equilibria under periodic harvesting," Mathematics Magazine, vol. 57, no. 3, pp. 156-158, 1984.

[17] P. Liu, J. Shi, and Y. Wang, "Periodic solutions of a logistic type population model with harvesting," Journal of Mathematical Analysis and Applications, vol. 369, no. 2, pp. 730-735, 2010.

[18] M. N. Nkashama, "Dynamics of logistic equations with nonautonomous bounded coefficients," Electronic Journal of Differential Equations, vol. 2000, pp. 1-8, 2000.

[19] S. Padhi, P. D. N. Srinivasu, and G. K. Kumar, "Periodic solutions for an equation governing dynamics of a renewable resource subjected to Allee effects," Nonlinear Analysis. Real World Applications, vol. 11, no. 4, pp. 2610-2618, 2010.

[20] E. Braverman and R. Mamdani, "Continuous versus pulse harvesting for population models in constant and variable environment," Journal of Mathematical Biology, vol. 57, no. 3, pp. 413-434, 2008.

[21] C. Castilho and P. D. N. Srinivasu, "Bio-economics of a renewable resource in a seasonally varying environment," Mathematical Biosciences, vol. 205, no. 1, pp. 1-18, 2007.

[22] M. Fan and K. Wang, "Optimal harvesting policy for single population with periodic coefficients," Mathematical Biosciences, vol. 152, no. 2, pp. 165-177, 1998.

[23] C. Xu, M. S. Boyce, and D. J. Daley, "Harvesting in seasonal environments," Journal of Mathematical Biology, vol. 50, no. 6, pp. 663-682, 2005.

[24] K. Gopalsamy, Stability and Oscillations in Delay Differential Equations of Population Dynamics, vol. 74 of Mathematics and Its Applications, Kluwer Academic Publishers, Dordrecht, The Netherlands, 1992.

[25] B. G. Zhang and K. Gopalsamy, "Global attractivity and oscillations in a periodic delay-logistic equation," Journal of Mathematical Analysis and Applications, vol. 150, no. 1, pp. 274283, 1990.

[26] K. Gopalsamy, X. Z. He, and L. Z. Wen, "Global attractivity and oscillations in a periodic logistic integrodifferential equation," Houston Journal of Mathematics, vol. 17, no. 2, pp. 157-177, 1991.

[27] K. Gopalsamy, X. Z. He, and L. Z. Wen, "On a periodic neutral logistic equation," Glasgow Mathematical Journal, vol. 33, no. 3, pp. 281-286, 1991.

[28] C. W. Clark, Mathematical Bioeconomics. The Mathematics of Conservation, Pure and Applied Mathematics, John Wiley \& Sons, Hoboken, NJ, USA, 3rd edition, 2010. 
[29] F. Brauer and D. A. Sánchez, "Constant rate population harvesting: equilibrium and stability," Theoretical Population Biology, vol. 8, no. 1, pp. 12-30, 1975.

[30] M. F. Laham, I. S. Krishnarajah, and J. M. Shariff, "Fish harvesting management strategies using logistic growth model," Sains Malaysiana, vol. 41, pp. 171-177, 2012.

[31] N. Keesom, T. Macrae, A. Uhlig, and R. Wang, "Fishing for answers: investigating sustainable harvesting rate models," Report of TARNADO Environmental Group, May 2010.

[32] J. H. Hubbard and B. H. West, Differential Equations: A Dynamical Systems Approach, Part I: Ordinary Differential Equations, Springer, New York, NY, USA, 2nd corrected edition, 1995.

[33] S. Wiggins, Introduction to Applied Nonlinear Dynamical Systems and Chaos, vol. 2 of Texts in Applied Mathematics, Springer, New York, NY, USA, 2nd edition, 2003.

[34] A. Berger and S. Siegmund, "Uniformly attracting solutions of nonautonomous differential equations," Nonlinear Analysis. Theory, Methods \& Applications, vol. 68, no. 12, pp. 3789-3811, 2008.

[35] J. K. Hale and H. Koçak, Dynamics and Bifurcations, vol. 3 of Texts in Applied Mathematics, Springer, New York, NY, USA, 1991.

[36] D. A. Sánchez, "Periodic environments, harvesting, and a Riccati equation," in Nonlinear Phenomena in Mathematical Sciences, V. Laksmikantham, Ed., pp. 883-886, Academic Press, New York, NY, USA, 1982.

[37] B. D. Coleman, Y. H. Hsieh, and G. P. Knowles, "On the optimal choice of $r$ for a population in a periodic environment," Mathematical Biosciences, vol. 46, no. 1-2, pp. 71-85, 1979.

[38] D. A. Sánchez, "A note on periodic solutions of Riccati-type equations," SIAM Journal on Applied Mathematics, vol. 17, pp. 957-959, 1969.

[39] V. A. Pliss, Nonlocal Problems of the Theory of Oscillations, Academic Press, New York, NY, USA, 1966.

[40] D. A. Sánchez, Ordinary Differential Equations: A Brief Eclectic Tour, Mathematical Association of America, Washington, DC, USA, 2002.

[41] J. L. Massera, "The existence of periodic solutions of systems of differential equations," Duke Mathematical Journal, vol. 17, pp. 457-475, 1950.

[42] F. B. Rizaner and S. P. Rogovchenko, "Dynamics of a single species under periodic habitat fluctuations and Allee effect," Nonlinear Analysis. Real World Applications, vol. 13, no. 1, pp. 141-157, 2012.

[43] M. Rasmussen, "Nonautonomous bifurcation patterns for one-dimensional differential equations," Journal of Differential Equations, vol. 234, no. 1, pp. 267-288, 2007. 


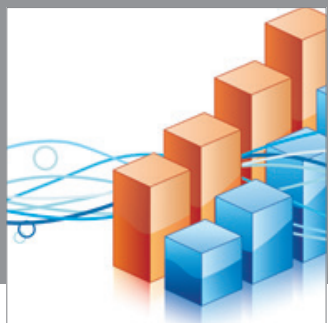

Advances in

Operations Research

mansans

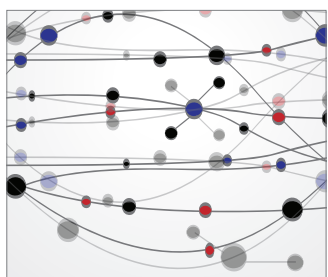

The Scientific World Journal
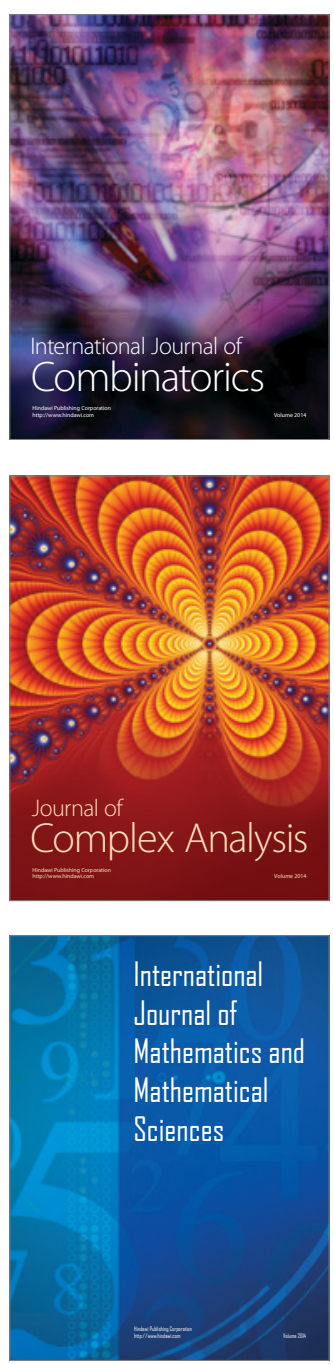
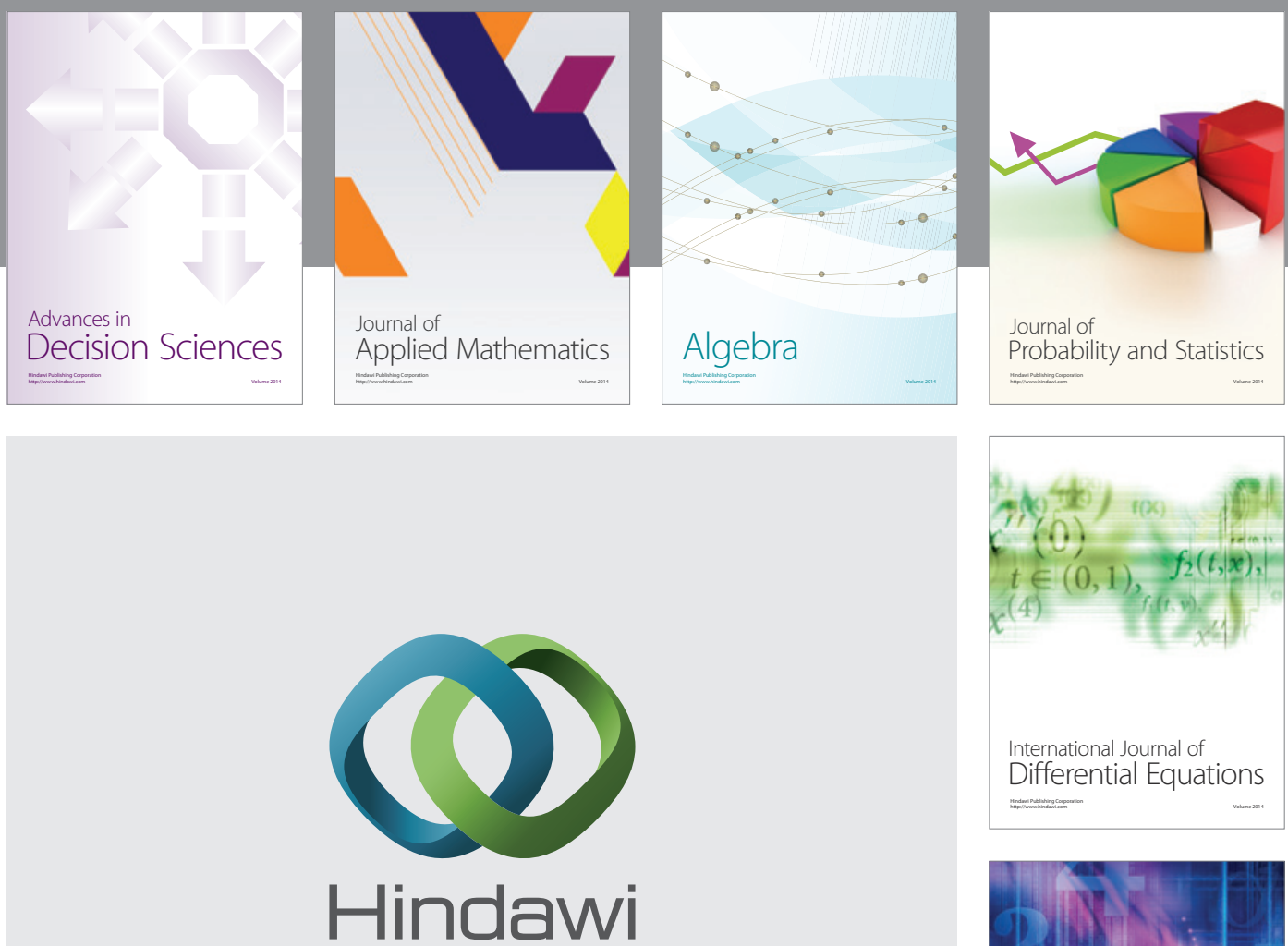

Submit your manuscripts at http://www.hindawi.com
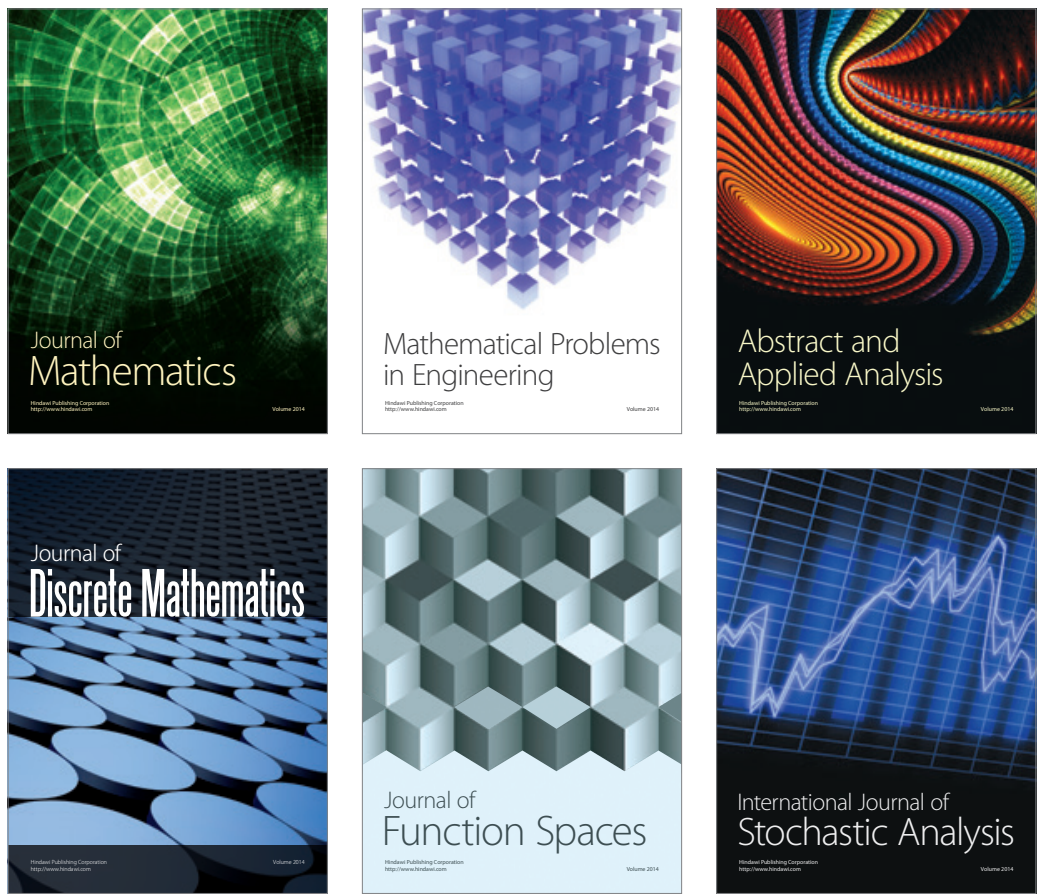

Journal of

Function Spaces

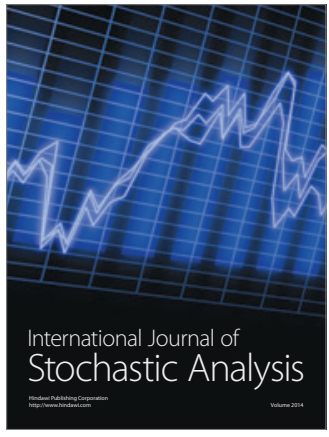

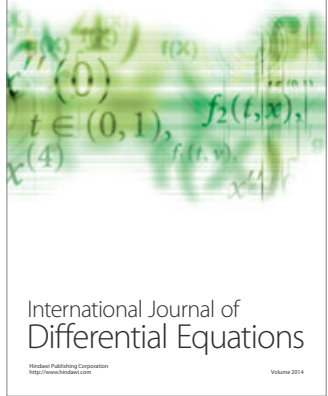
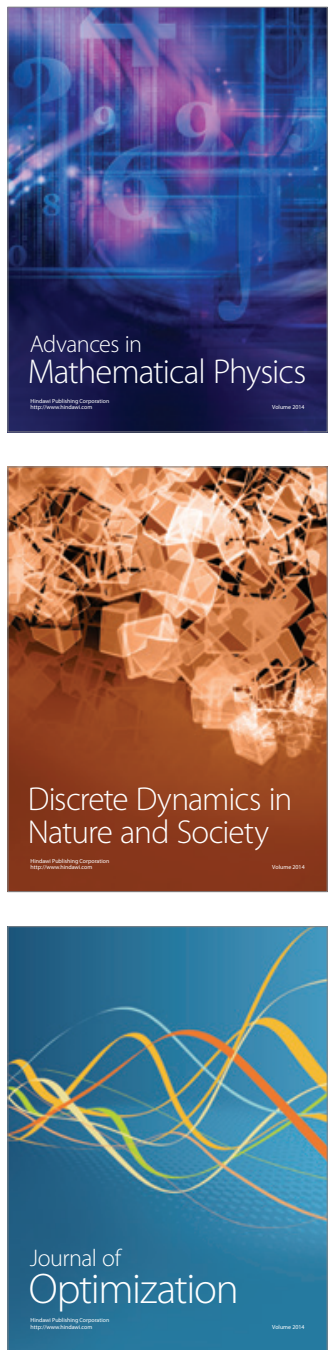